\title{
Gradient-Based Propeller Optimization with Acoustic Constraints
}

\author{
Daniel Ingraham* and Justin Gray ${ }^{\dagger}$ \\ NASA Glenn Research Center, Cleveland, Ohio, 44135 \\ Leonard V. Lopes \\ NASA Langley Research Center, Hampton, Virginia, 23681
}

\begin{abstract}
Urban Air Mobility vehicles are intended to operate near or within large cities, where a significant portion of the public will be exposed to the noise they create. If these vehicles are to become acceptable to the public, designers must be able to manage the amount of noise they generate, and understand the relationship between traditional performance metrics (thrust, efficiency, etc.) and noise. As a first step to addressing this need, this work combines a blade element momentum theory tool (OpenBEMT) with an acoustic prediction tool (ANOPP2) to optimize a propeller subject to both aerodynamic and acoustic constraints. These tools are developed within a optimization framework (OpenMDAO) that allows analytic derivatives to be propagated through the models and passed to a gradient-based optimizer. This toolchain is exercised on the cruise propellers from the X-57 Maxwell, and yields propeller designs that reduced the overall sound pressure level by about $5 \mathrm{~dB}$ for a cost of $1 \%$ propeller efficiency.
\end{abstract}

\section{Nomenclature}

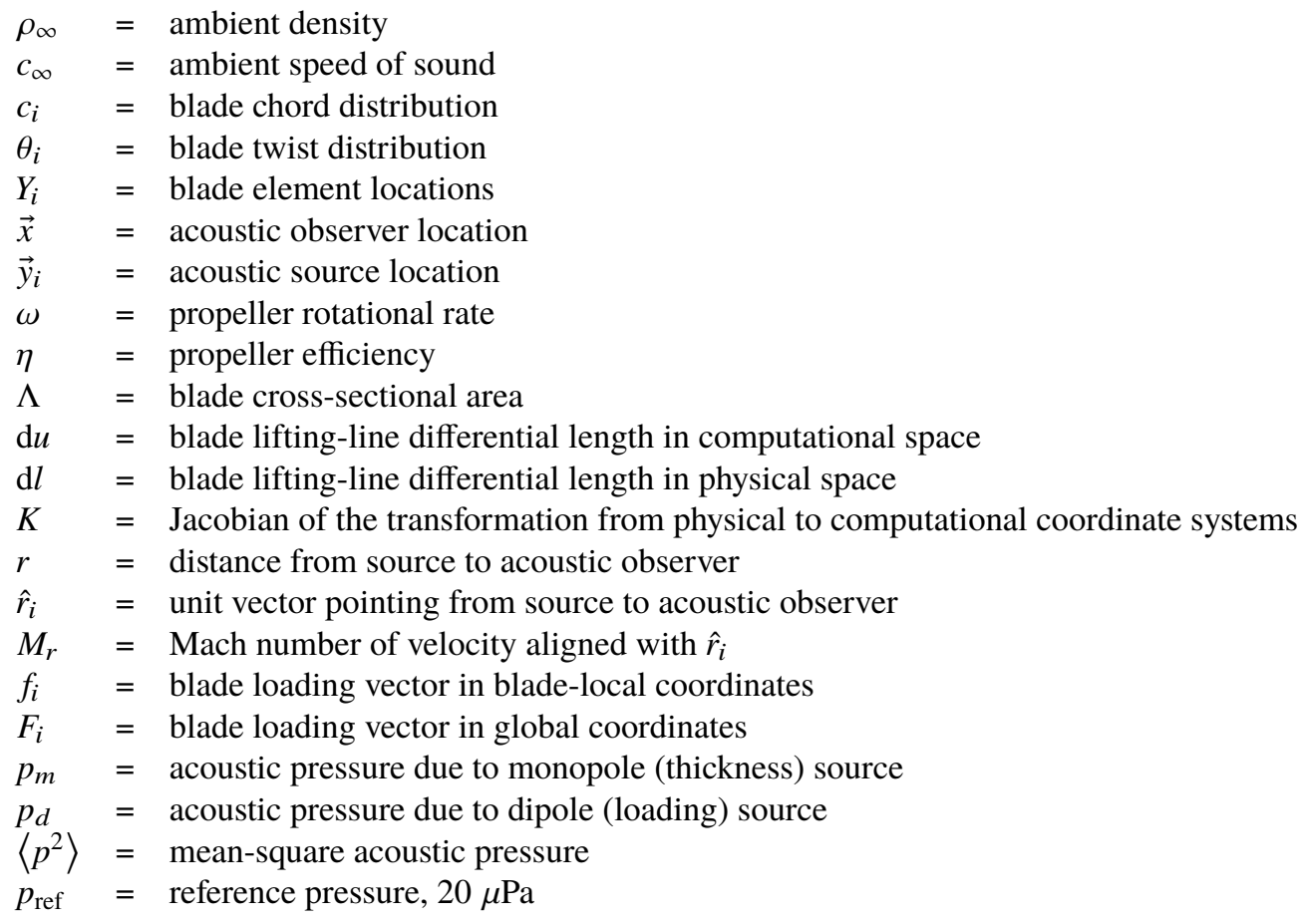

\footnotetext{
*Research Aerospace Engineer, Acoustics Branch, MS 54-3, Member AIAA.

${ }^{\dagger}$ Research Aerospace Engineer, Propulsion Systems Analysis Branch, MS 5-11, Member AIAA.

$¥$ Research Aerospace Engineer, Aeroacoustics Branch, MS 461, Member AIAA.
} 


\section{Introduction}

Recently, the Urban Air Mobility (UAM) concept has become a popular research topic in the aerospace community. In this concept, small, likely electric-powered vehicles will be used to move people and goods within or near large cities. If such an idea is to gain any traction, the noise produced by these vehicles must be acceptable to the public, and engineers must have access to design tools that allow them to trade e.g. aerodynamic performance for noise.

A number of examples of propeller optimization with acoustic constraints can be found in the literature. Miller and Sullivan[1] combined a vortex lattice aerodynamic model with a subsonic point source acoustic model to optimize propeller twist, chord, and sweep distributions for both noise and performance. Gur and Rosen[2] combined aerodynamic, structural, and acoustic models while analysing and optimizing a propeller for a small, electric-powered UAV, and later [3] incorporated additional components of the vehicle (engine, gear box, etc.). Pagano et al.[4, 5] performed an impressive optimization of a propeller in a pusher configuration through the coupling of a computational fluid dynamics and computational structural dynamics code. The design variables in this work included not only the shape of the blade, but also the position of the engine exhaust ducts, as the non-uniform flow created by the ducts influences the propeller performance and noise. Finally, Wisniewski et al.[6] used blade element momentum theory and the National Renewable Energy Laboratory's NAFNOISE code to optimize small propellers intended for hand-launched unmanned aircraft.

In this work, two existing tools, OpenBEMT and ANOPP2[7], are combined to study the relationship between the aerodynamic performance and acoustics of the cruise propeller from the X-57 Maxwell vehicle. These two tools are tied together using OpenMDAO[8], an open-source multidisciplinary design, analysis, and optimization framework. This work represents a first step towards performing a comprehensive optimization study of an urban air mobility vehicle that will include additional disciplines (trajectory, power generation, aerodynamics, etc.). The ultimate goal of this effort is to provide the tools necessary for the larger UAM vehicle optimization to include a constraint on a noise metric over the entire trajectory. This will require including the optimization presented here at each vehicle trajectory point, propagating the noise to an observer on the ground, and aggregating the observed noise through the evaluation of an appropriate acoustic metric. Isolating and implementing the propeller performance and noise prediction components of that process is the focus here.

\section{Propeller Model}

\section{A. Aerodynamics}

The propeller aerodynamics are predicted here using blade element momentum theory (BEMT). BEMT begins by dividing the blade spanwise into a finite number of radial stations, or "blade elements." Each blade element is treated as an independent airfoil with associated lift and drag curves. A control volume is then formed that encloses the propeller and the flow far upstream and downstream. By applying a momentum balance to this control volume, and including the interaction between the fluid and blade elements, the velocity induced by the propeller in the axial and circumferential directions can be predicted. The induced velocities can then be used to calculate desired propeller performance metrics, such as the necessary input power, the net torque and thrust, and propeller efficiency.

The BEMT implementation used here is OpenBEMT, first described by Hwang and Ning[9]. In OpenBEMT, information from an airfoil database (wWw.airfoiltools.com) is extended past stall using the Viterna method[10] and minimal-energy tensor-product splines as implemented in the Surrogate Modeling Toolbox[11]. The code also uses a modification to traditional BEMT that reduces the method to the solution of a single implicit equation[12]. OpenBEMT uses the OpenMDAO framework $[\overline{8}$ to propagate both the outputs and their derivatives through each stage of the BEMT process. OpenMDAO then aggregates the derivative information and passes it to an optimizer, using it in the gradient-based optimization process. OpenBEMT can be used as a stand-alone propeller design tool, giving an optimized propeller design subject to constraints specified by the user. Here, it is used to predict the load distribution on the propeller blades, which is an input to the noise prediction method, described below.

Blade element momentum theory assumes that there is no interaction between radial stations, and is thus not suitable for blades with significant spanwise flow. The propeller blades considered in this work are unswept and rather slender, making BEMT an appropriate choice. The particular form of blade element momentum theory currently implemented in OpenBEMT assumes steady, level flight with the propeller shaft axis aligned with the incoming flow. This implies that the loads predicted by OpenBEMT will be steady, i.e., they will not be a function of azimuthal position or time. As discussed in the next section, this limits the acoustic sources that can be captured by the noise prediction code to so-called "steady" sources. Fortunately, steady propeller noise sources are expected to be the most significant for the configuration considered in this work[13]. 


\section{B. Acoustics}

The noise radiated by the propeller is predicted using an acoustic analogy, specifically Farrasat's 1A formulation[14, 15] of the Ffowcs-Williams Hawking (FWH) approach[16]. Like all acoustic analogies, the FWH method begins with the Navier-Stokes equations, and algebraically manipulates them into a propagation term and one or more source terms. In the case of the FWH equation, the propagation term is the unsteady wave equation for acoustic pressure, and three source terms are identified: the monopole, dipole, and quadrupole terms. The FWH equation is solved using generalized functions and the free-space Green's function for the wave equation, which require the evaluation of a surface integral of the monopole and dipole terms, and a volume integral of the quadrupole term. In propeller and rotor acoustic analysis, the integration surface is often taken as the blade surface, and the volume integral associated with the quadrupole term is ignored. Farrasat's F1A formulation, the form of the FWH approach used here, is algebraically equivalent to the original FWH method, but switches the order of the surface integral and a temporal derivative, which is numerically beneficial.

A compact version Farrasat's 1A formulation of the FWH is used in this work: the integration surface (here, the propeller blade) usually associated with the method is replaced with a radial lifting line on which the net aerodynamic forces at each radial station act. The compact form of the F1A equation assumes that the original integration surface is elongated and that the acoustic observer distance is much larger than the characteristic thickness of the blade—both of these criteria are likely to be satisfied by urban air mobility concept vehicles. Besides reducing the computation cost of the non-compact F1A method, the inputs needed for the compact F1A calculation directly correspond to the outputs provided by blade element momentum theory.

The details of the compact F1A method used here are described by Lopes[17]. Briefly, the solution for the monopole term is

$$
\frac{4 \pi}{\rho_{\infty}} p_{m}=\int\left[(\ddot{\Lambda} K+2 \dot{\Lambda} \dot{K}+\Lambda \ddot{K}) A_{1 \mathrm{~A}}+(\dot{\Lambda} K+\Lambda \dot{K}) B_{1 \mathrm{~A}}+(\Lambda K) C_{1 \mathrm{~A}}\right]_{\mathrm{ret}} \mathrm{d} u,
$$

and, for the dipole term,

$$
4 \pi c_{\infty} p_{d}=\int\left[\left(\dot{F}_{i} K+F_{i} \dot{K}\right) D_{1 \mathrm{~A}, \mathrm{i}}+\left(F_{i} K\right) E_{1 \mathrm{~A}, i}\right]_{\mathrm{ret}} \mathrm{d} u .
$$

The factors $A_{1 \mathrm{~A}}, B_{1 \mathrm{~A}}, C_{1 \mathrm{~A}}, D_{1 \mathrm{~A}}$, and $E_{1 \mathrm{~A}}$ are functions of the motion of the lifting line, and do not depend on the local loading $F_{i}$ or cross-sectional area $\Lambda$. The ret subscript indicates the bracketed quantities are evaluated at the retarded time, and the dots indicate a derivative with respect to the retarded time.

In this work, the blade cross-sectional area and the magnitude of the loads acting on the propeller blades are only a function of the radial distance along the blade - they do not vary in time, or with the propeller rotation. This simplifies the F1A equation considerably, and gives, for the monopole term (1),

$$
\frac{4 \pi}{\rho_{\infty}} p_{m}=\int\left[\Lambda C_{1 \mathrm{~A}}\right]_{\mathrm{ret}} \mathrm{d} l,
$$

where

$$
C_{1 \mathrm{~A}}=R(0,2) \ddot{R}(1,1)+R(0,1) \dot{R}(0,1) \dot{R}(1,1)
$$

and

$$
R(n, m)=r^{-n}\left(1-M_{r}\right)^{-m} .
$$

Equation (3) shows, for the assumptions used in this work, the simple relationship between the blade cross-sectional area $\Lambda$ and the acoustic pressure: an increase in cross-sectional area will increase $p_{m}$, especially in regions of high blade velocity.

For the dipole term in equation (2), assuming constant loading gives

$$
4 \pi c_{\infty} p_{d}=\int\left[\dot{F}_{i} D_{1 \mathrm{~A}, \mathrm{i}}+F_{i} E_{1 \mathrm{~A}, i}\right]_{\mathrm{ret}} \mathrm{d} l,
$$

where

$$
\begin{gathered}
D_{1 \mathrm{~A} i}=R(0,1) B_{1 i}, \\
E_{1 \mathrm{~A} i}=R(0,1) \dot{B}_{1 i}+c_{\infty} R(2,1) \hat{r}_{i},
\end{gathered}
$$

and

$$
B_{1 i}=R(1,1) \hat{r}_{i} .
$$




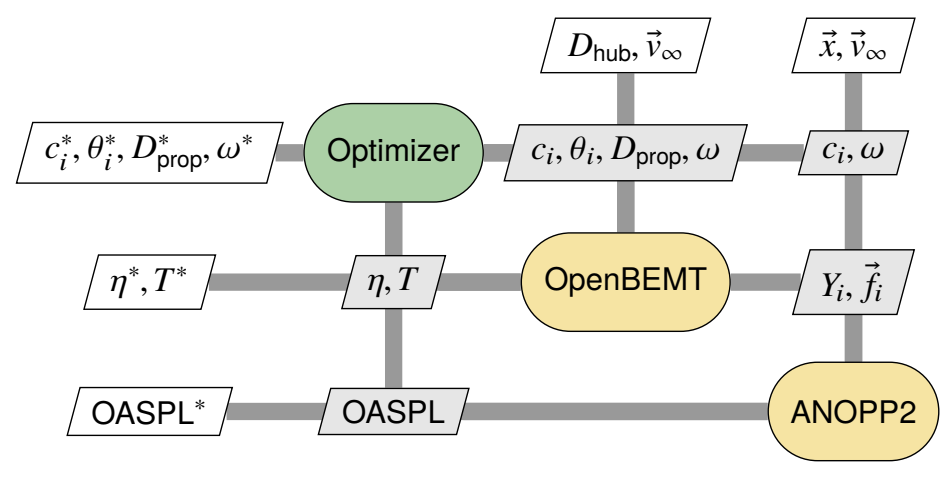

Fig. 1 Extended Design Structure Matrix (XDSM) diagram for the optimization.

Similar to the monopole term, equation (6) shows a simple relationship between the loading $F_{i}$ and the predicted acoustic pressure $p_{d}$ : at a given radial station, the magnitude of $p_{d}$ will increase with increasing load, especially in regions where the blade is moving quickly.

The compact F1A technique is implemented in ANOPP2[7], NASA Langley's second generation Advanced Noise Prediction Program. ANOPP2's compact F1A includes the derivatives of the F1A output (the time history of acoustic pressure) with respect to the flow state along the lifting line, and also derivatives of the acoustic metric routines (e.g., overall sound pressure level) with respect to their inputs. These derivatives are provided to the OpenMDAO framework during the optimization process, which are in turn passed by OpenMDAO to the gradient-based optimization routine.

Because OpenBEMT currently is restricted to assuming constant loading for steady, level flight, there are a number of noise sources that will not be captured by the compact F1A approach taken here. Unsteady sources, such as when the propeller shaft is not aligned with the flow direction or when the inflow velocity is non-uniform, cannot be predicted. Broadband noise, such as that caused by inflow turbulence or a turbulent boundary layer, will also not be present in these predictions. The sources that are accounted for here (the "thickness" and "loading" noise associated with the monopole and dipole terms of the F1A, respectively) are the most significant propeller noise sources for full-scale propellers undergoing steady, level flight[13], and should be adequately predicted by OpenBEMT and ANOPP2.

\section{Optimization Approach}

The blade element momentum theory of OpenBEMT and the compact F1A implementation of ANOPP2 are combined into an propeller optimization code with the OpenMDAO [8] framework. OpenMDAO is an open-source Python implementation of the modular analysis and unified derivatives[18] (MAUD) approach to multidisciplinary design, analysis, and optimization (MDAO). OpenMDAO allows the application developer to express an analysis as a connected series of components, where each component uses inputs to calculate not only the outputs, but also the derivatives of the outputs with respect to the inputs. It then assembles these partial derivatives together into a total derivative, i.e., the derivative of the problem objective and constraints with respect to the design variables. These total derivatives are passed by OpenBEMT to a gradient-based optimizer to find the solution to the optimization problem. The OpenMDAO framework is used here to couple together the OpenBEMT and ANOPP2 codes, and the pyOptSparse Python package is used to call SNOPT[19], a sparse nonlinear optimizer.

A simplified Extended Design Structure Matrix showing the relationship between OpenBEMT and ANOPP2 is found in Figure 1 . The $i$ subscripts indicate a variable that varies along the blade length, and the $*$ superscripts indicate the final optimized values returned by the optimizer. OpenBEMT takes information describing the shape and motion of the propeller and calculates aerodynamic performance metrics such as thrust and efficiency. It also returns the $\vec{f}_{i}$, the propeller loading in a coordinate system attached to the blade, and $Y_{i}$, the blade element locations in the same coordinate system. ANOPP2 uses this information to calculate the acoustic pressure experienced by an observer located at $\vec{x}$, and then calculates an acoustic metric-here, the overall sound pressure level (OASPL).

The XDSM for the ANOPP2 block in Figure1 1 is expanded in Figure 2 Two preprocessing components, "Blade Geometry" and "Blade Loads," calculate the motion and loading of each blade, along with the cross-sectional area of each blade element, necessary for the monopole term in (3). The modular structure of ANOPP2 makes the acoustic 


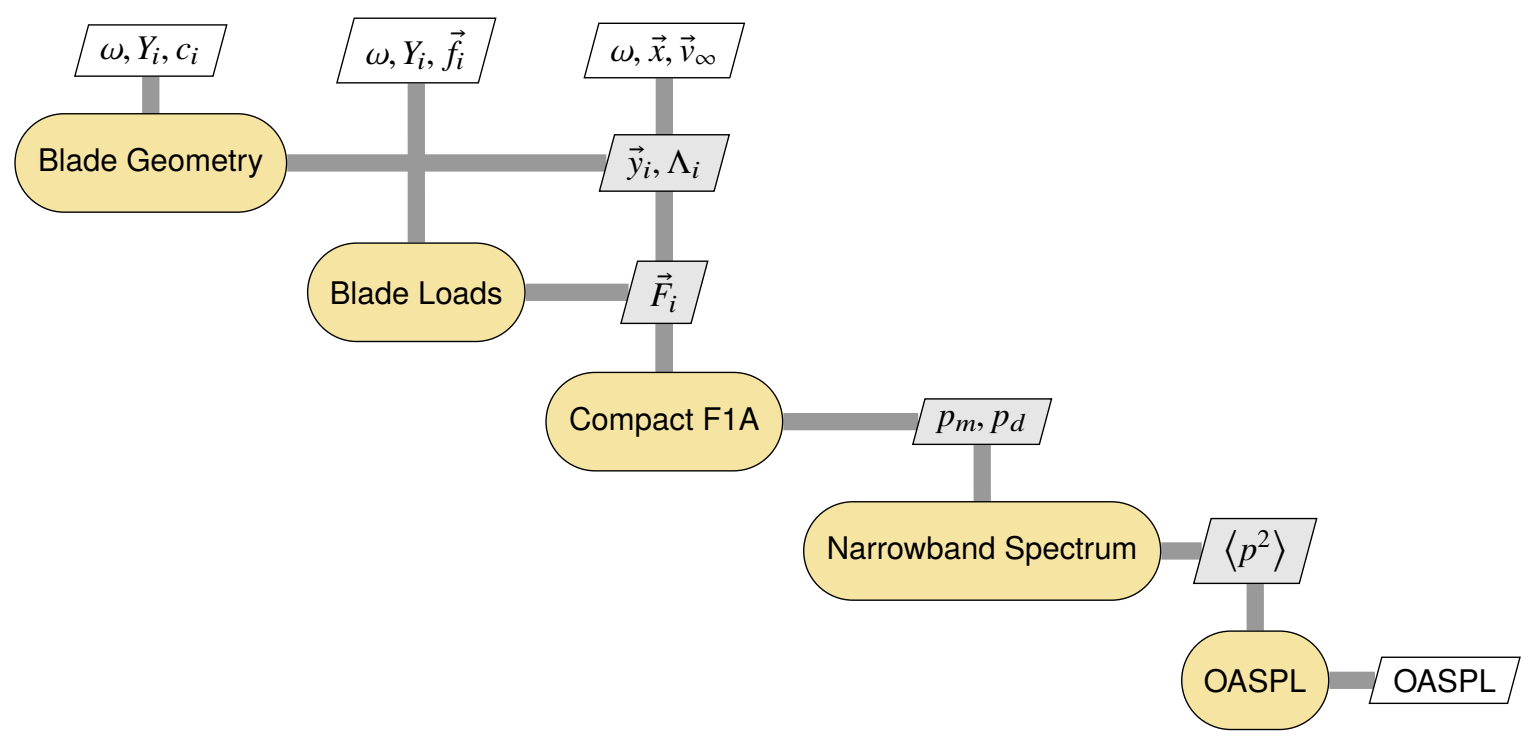

Fig. 2 Extended Design Structure Matrix (XDSM) diagram for the ANOPP2 portion of the optimization.

\begin{tabular}{ll}
\hline Property & Value \\
\hline Altitude & $10 \mathrm{~m}$ \\
Cruise speed & $77.2 \mathrm{~m} / \mathrm{s}$ \\
Propeller rotation rate & $236 \mathrm{rad} / \mathrm{s}$ \\
Diameter & $1.5 \mathrm{~m}$ \\
Hub diameter & $0.3 \mathrm{~m}$ \\
Airfoil & MH117 \\
Blade count & 3 \\
\hline
\end{tabular}

Table 1 X-57 cruise propeller properties.

analysis quite flexible-for example, the compact F1A component could be replaced with a non-compact version if the loading distribution in the chordwise and thickness directions was available from, say, a computational fluid dynamics calculation. The OASPL calculation could also be replaced with any of the many other acoustic metrics available in ANOPP2.

Currently, ANOPP2 provides derivatives of the outputs of each component shown in Figure 2 with respect to all inputs, with the exception of $\omega$, the rotational rate of the propeller. For this work, these derivatives were computed using a module external to the ANOPP2 library, which allowed $\omega$ to be used as a design variable during the optimization.

\section{Discussion of Results}

Settings for the propeller optimization case presented in this work were taken from the X-57 cruise propellers in Hwang and Ning's recent paper[9]. The X-57 geometric parameters are summarized in Table 1

\section{A. Optimization Case 1}

The parameters for the first optimization case are presented in Table 2 For this case, the objective was to maximize propeller efficiency, subject to a total thrust constraint of $700 \mathrm{~N}$ and a change in sideline overall sound pressure level 


\begin{tabular}{cllll}
\hline & Variable & Lower Bound & Upper Bound & Count \\
\hline maximize & efficiency & & & \\
& & & & \\
with respect to & chord distribution & $1 \mathrm{~cm}$ & $50 \mathrm{~cm}$ & 15 radial stations \\
& twist distribution & $20 \mathrm{deg}$ & $90 \mathrm{deg}$ & 15 radial stations \\
subject to & total thrust & $700 . \mathrm{N}$ & $700 . \mathrm{N}$ & 1 \\
& sideline OASPL, 10 m from hub & $x \Delta \mathrm{dB}$ & $x \Delta \mathrm{dB}$ & 1 \\
\hline
\end{tabular}

Table 2 Parameters for optimization test case 1. The OASPL value $x$ is referenced to the optimization with no noise constraint, and was systematically decreased from 0 to form a Pareto frontier.

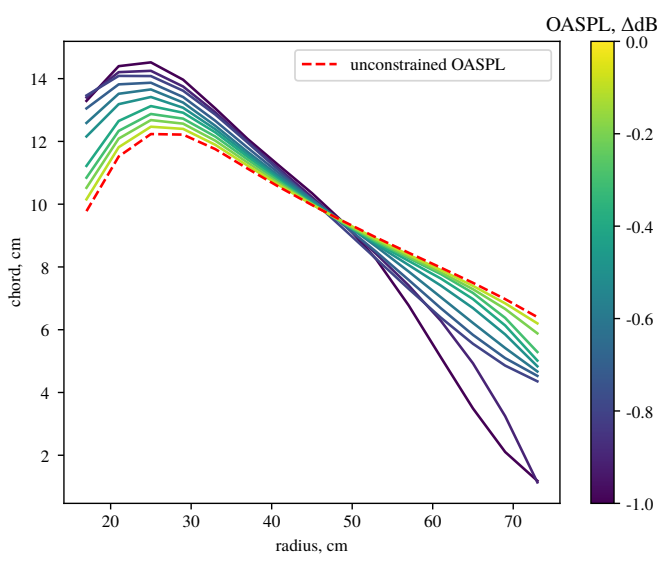

(a) Blade chord distribution

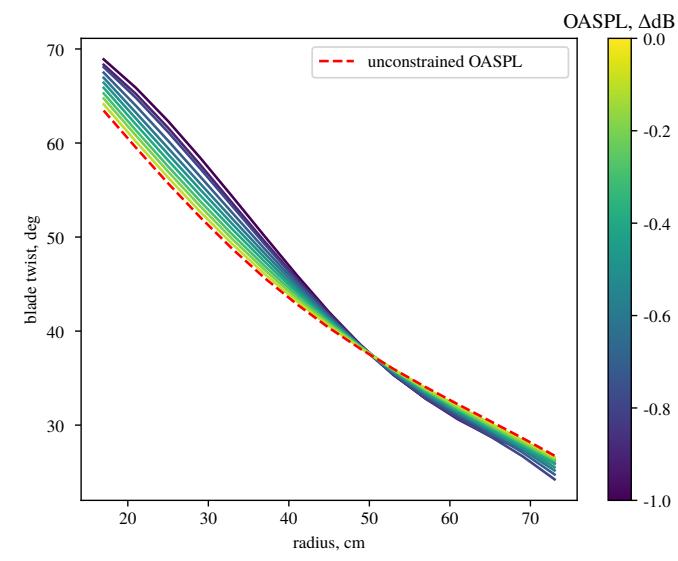

(b) Blade twist distribution

Fig. 3 Design variable results for case 1.

(OASPL)

$$
\text { OASPL }=10 \log _{10}\left(\frac{\left\langle p^{2}\right\rangle}{p_{\text {ref }}^{2}}\right)
$$

measured 10 meters from the propeller hub that ranged from 0 to $-1 \mathrm{~dB}$. This OASPL value is referenced to the optimization with no noise constraint.

Figures $3 \mathrm{a}$ and $3 \mathrm{~b}$ show the chord and twist of the blade, respectively, for the range of OASPL constraints considered, along with the results for the OASPL unconstrained. (The $x$-axis is the location of each blade element's center point, so the distribution does not quite extend to the hub and tip radius values of 15 and 75 centimeters.) As the OASPL constraint is decreased, both the chord and twist distribution is gradually shifted inward, toward the hub of the propeller. The chord distributions for $\triangle$ OASPL constraints of -0.9 and $-1.0 \Delta \mathrm{dB}$ appear to bottom out at the lower limit of $1 \mathrm{~cm}$ at the blade tip. As explained in Section II.B, a radial station's contribution to the thickness noise is a function of the cross-sectional area at that station, which in this work is determined by the chord. Reducing the chord at the blade tip, where the velocity is the highest, will significantly reduce the thickness noise. Likewise, since a radial station's loading is directly related to the blade twist, the movement of twist from tip to hub reduces loading noise.

Figures $4 \mathrm{a}$ and $4 \mathrm{~b}$ show loading in the axial and circumferential directions along the blade, respectively. The changes in the blade geometry shown in Figures $3 a$ and $3 b$ have shifted the axial load from the blade tip to the midsection for the OASPL-constrained cases. A similar effect is seen in the circumferential loading distribution, where most of the OASPL-constrained blades see a sharp increase in the circumferential load midspan, and a significant reduction near the blade tip. Since the loading noise is directly proportional to the load on the blades, and is strongest where the loads move quickly, this shift in loading from tip to midspan has the effect of decreasing the loading noise term in the F1A 


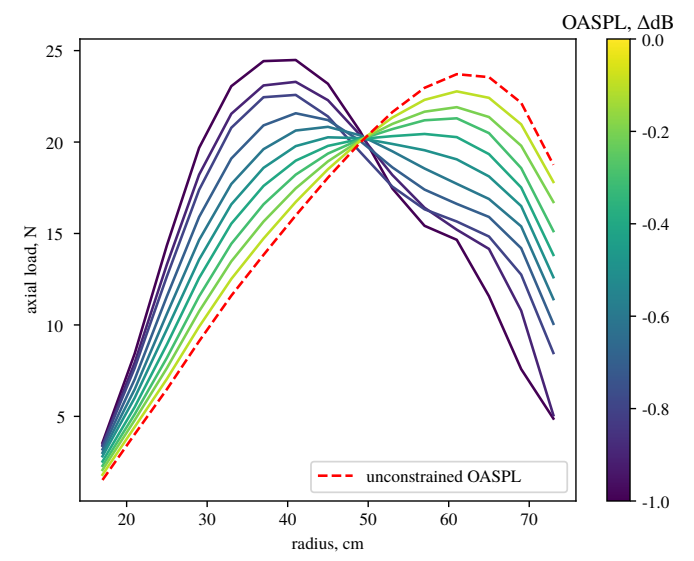

(a) Blade axial load distribution

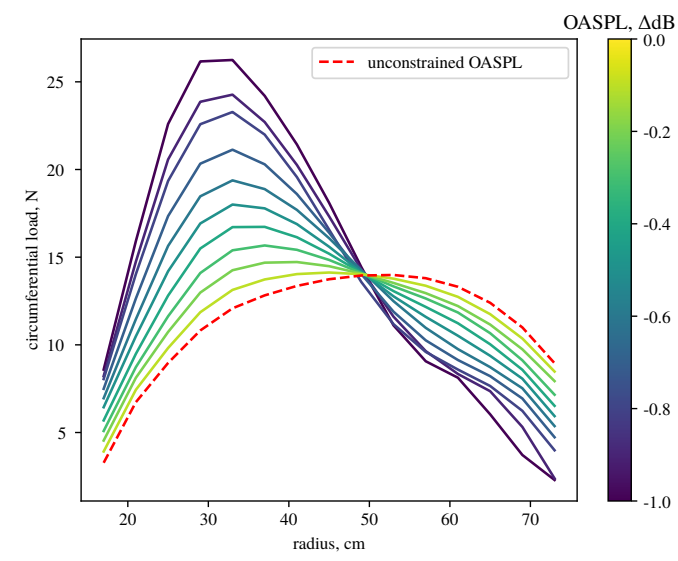

(b) Blade circumferential load distribution

Fig. 4 Blade loading results.

\begin{tabular}{cllll}
\hline & Variable & Lower Bound & Upper Bound & Count \\
\hline maximize & efficiency & & & \\
& & & & \\
with respect to & chord distribution & $1 \mathrm{~cm}$ & $50 \mathrm{~cm}$ & 15 radial stations \\
& twist distribution & $20 \mathrm{deg}$ & $90 \mathrm{deg}$ & 15 radial stations \\
& propeller diameter & $75 \mathrm{~cm}$ & $225 \mathrm{~cm}$ & 1 \\
& propeller rotational rate & None & None & 1 \\
subject to & total thrust & $700 . \mathrm{N}$ & $700 . \mathrm{N}$ & 1 \\
& sideline OASPL, 10 m from hub & $x \Delta \mathrm{dB}$ & $x \Delta \mathrm{dB}$ & 1 \\
\hline
\end{tabular}

Table 3 Parameters for optimization test case 2. As in case 1, the OASPL value is referenced to the optimization with no noise constraint, and was systematically decreased from 0 to form a Pareto frontier.

calculation.

Figure 5 shows the relationship between the value of the OASPL constraint and the efficiency objective. Overall, an OASPL reduction of approximately $1 \Delta \mathrm{dB}$ is achieved for a $4 \%$ drop in propeller efficiency. Interestingly, the points for OASPL of 0.9 and $1.0 \Delta \mathrm{dB}$ (and the optimizations that ran up against the lower chord limit at the blade tip) don't quite follow the trend of the rest of the curve.

\section{B. Optimization Case 2}

The second optimization case considered in this work is identical to the first except for the addition of two design variables: the propeller diameter and rotational rate (see Table 3). As shown in Table 3, the propeller diameter design variable had an upper bound of $225 \mathrm{~cm}$. Since no structural model is included in this work, the optimizer chose the diameter upper bound for each run, regardless of the value of the OASPL constraint. Essentially, the upper limit of the propeller diameter design variable acted as a constraint. As might be expected, this larger diameter propeller is considerably more efficient than the original: OpenBEMT predicts $99.0 \%$ efficiency for the optimization without the acoustic constraint, compared to $95.2 \%$ for case 1 .

Figure 6 shows the propeller rotational rate design variable as a function of the OASPL constraint. The rotational rate is reduced in a nearly linear fashion as the noise constraint is tightened. This will obviously lower the speed of the entire blade, reducing the $R(n, m)$ terms in the compact F1A equations and thus the noise.

The chord and twist distributions for case 2 are shown in Figure 7 Both distributions differ considerably from the 


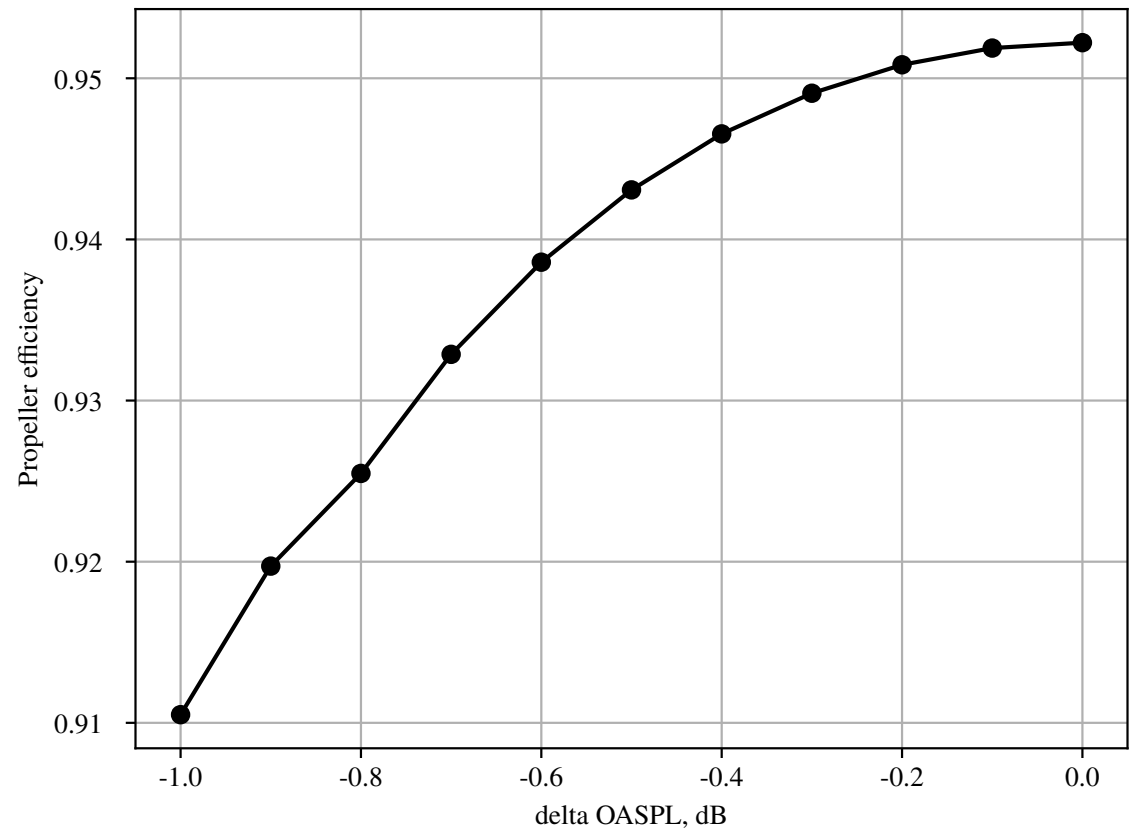

Fig. 5 Relationship between the noise constraint and efficiency objective for case 1.

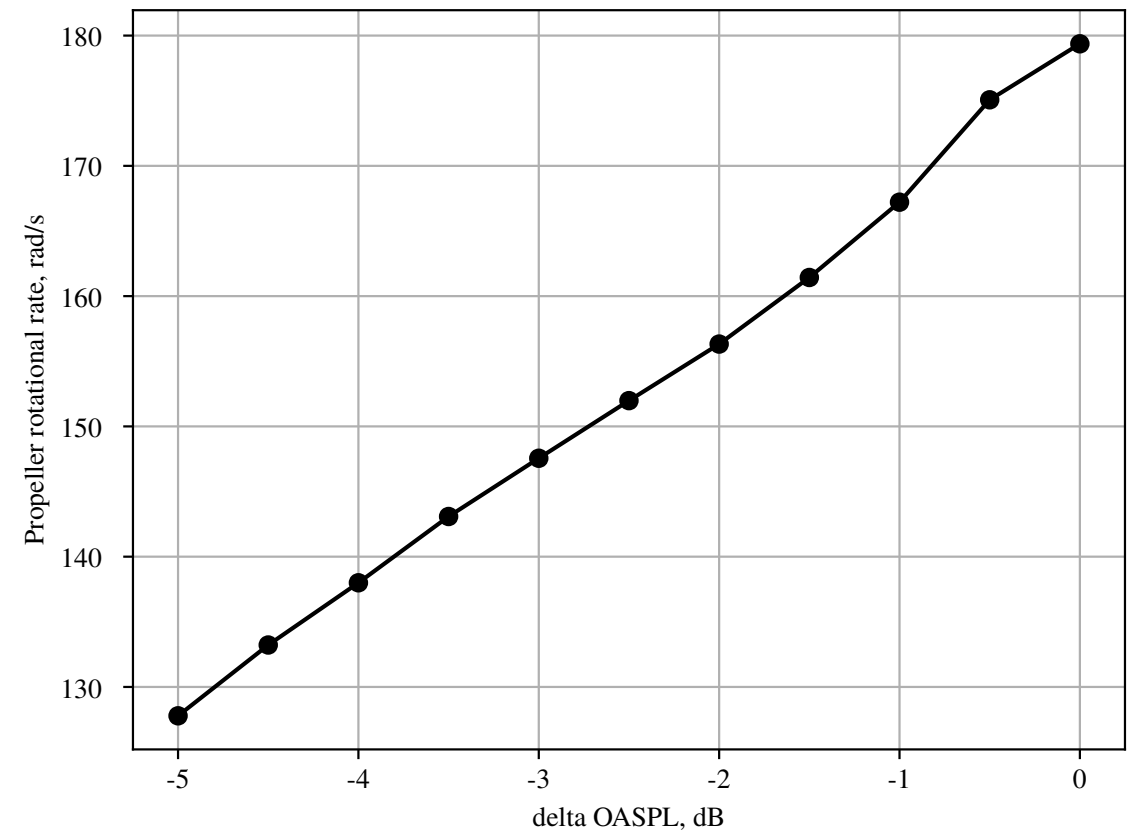

Fig. 6 Propeller rotational rate design variable results for case 2. 


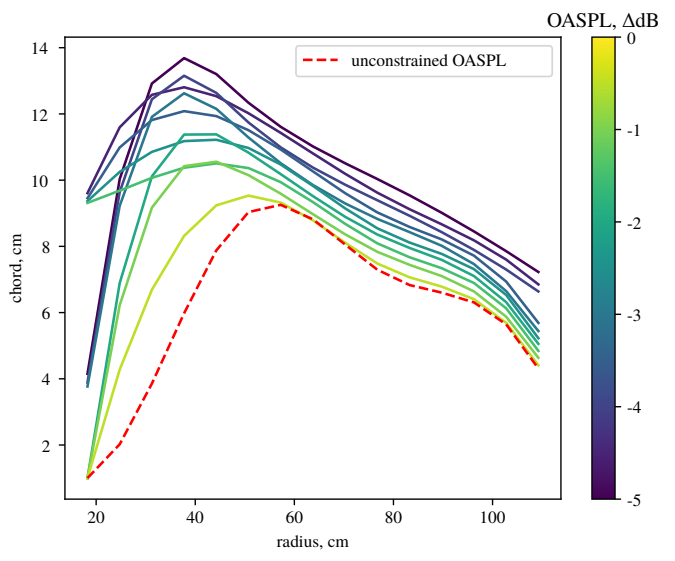

(a) Blade chord distribution

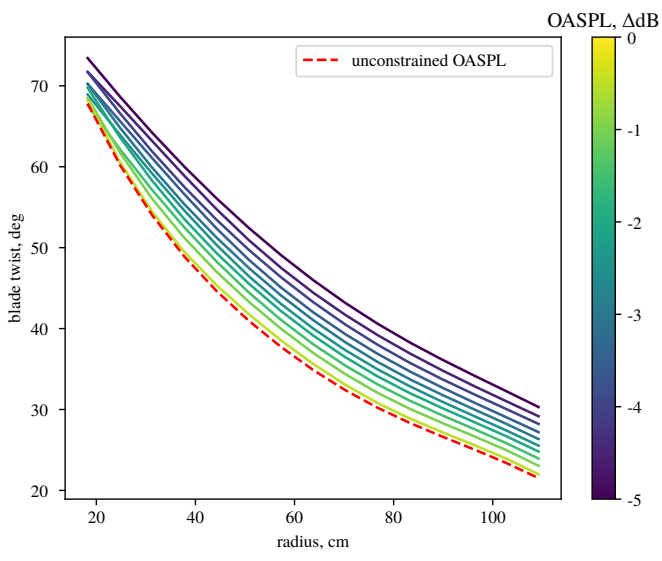

(b) Blade twist distribution

Fig. 7 Chord and twist design variable results for case 2.

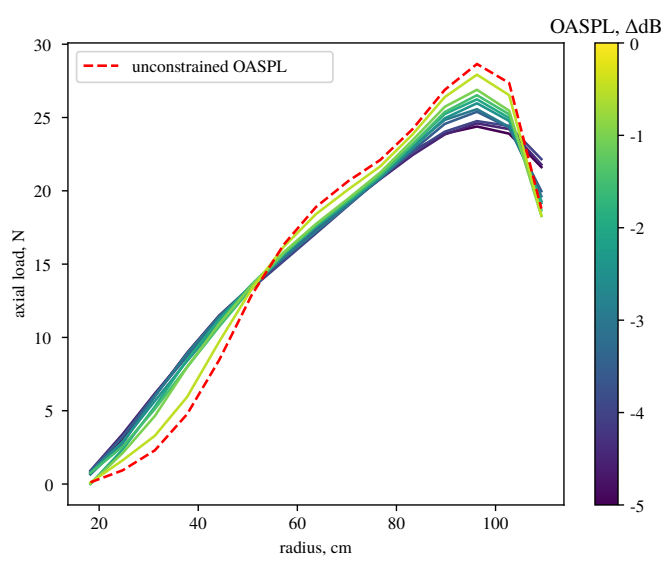

(a) Blade axial load distribution

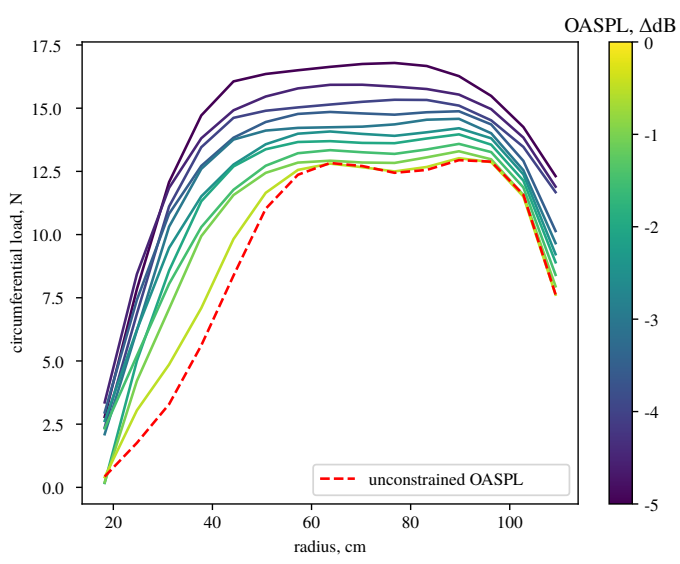

(b) Blade circumferential load distribution

Fig. 8 Blade loading results for case 2. 


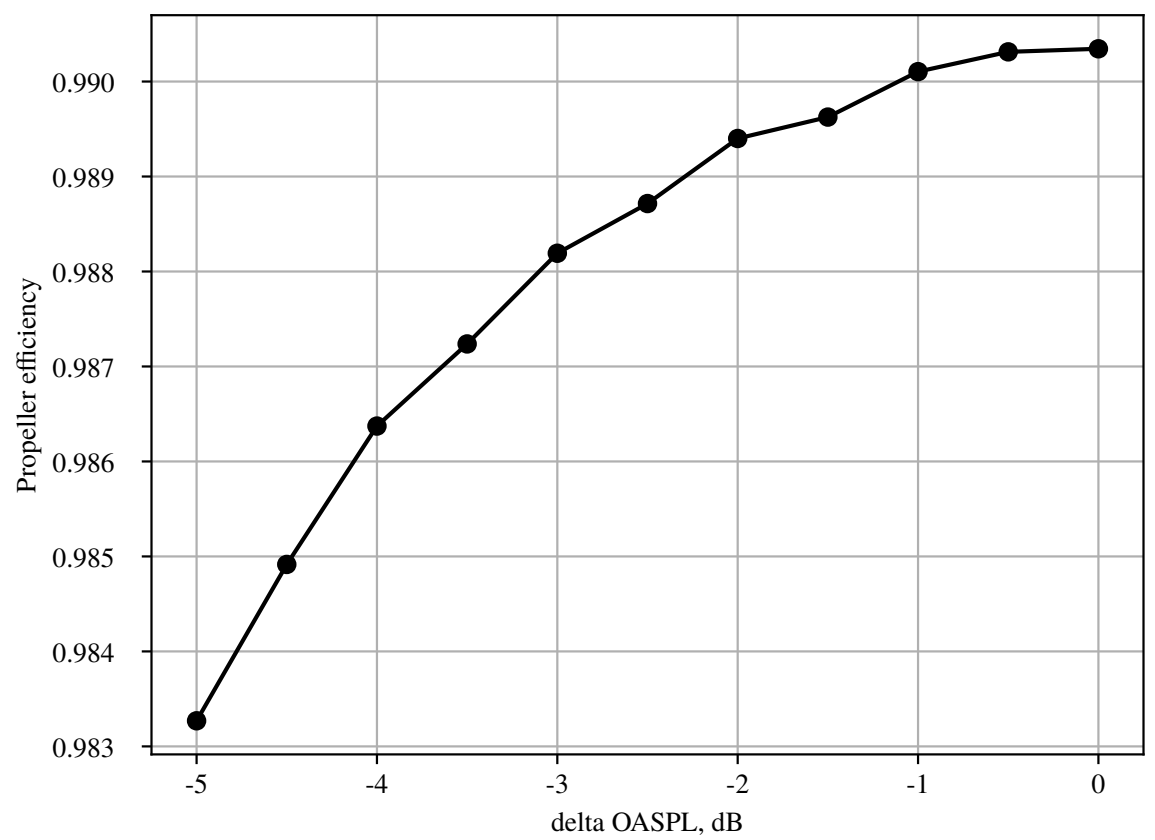

Fig. 9 Relationship between the noise constraint and efficiency objective for case 2.

previous case. Comparing these results to the corresponding plots for case 1 in Figure 3 shows how the optimizer has taken a different strategy for case 2: rather than shifting the chord and twist inward to the slower-moving region of the blade, it chooses to slow the entire propeller by reducing the rotational rate, while simultaneously increasing both the chord and twist to maintain thrust. The effect of this strategy on the blade loading is shown in Figure 8 Again, these results are quite unlike case 1 . By increasing the chord and twist as the rotational rate is reduced, the optimizer is able to maintain nearly the same axial load along the blade as the noise constraint is tightened. This comes at the cost of increasing the circumferential load, which reduces the propeller's efficiency.

Figure 9 plots the propeller efficiency as a function of the OASPL constraint value. The cost of lowering the propeller's noise is considerably reduced compared to case 1: an OASPL of $-5 \Delta \mathrm{dB}$ requires only a $1 \%$ reduction in propeller efficiency. Figure 10 shows the same data compared with an identical optimization, but with the propeller diameter upper limit increased from 2.25 meters to 3. Increasing this limit has the effect of shifting the Pareto front in the direction of higher efficiency.

\section{Conclusions}

In this work, a propeller performance code and a noise prediction code were combined within a MDAO framework to analyze and optimize a propeller design for performance, subject to a noise constraint. The objective of the optimization case was to maximize propeller efficiency, subject to a thrust equality constraint and a range of overall sound pressure levels (OASPL). The design variables were the chord and pitch distributions along each blade, and, for the second case, the diameter and rotational rate of the propeller. For the second optimization case considered, OASPL reductions of about $5 \mathrm{~dB}$ relative to the baseline case were achieved, at the cost of about a $1 \%$ reduction in the propeller's efficiency. When the propeller's diameter and rotational rate were fixed, the optimizer achieved noise reductions by shifting the chord and loading along the blade from outboard in, where the blade velocity is lower. When diameter and rotational rate were allowed to vary, the optimizer maximized the diameter value and gradually reduced the rotational rate to meet the tightening OASPL constraint.

The ultimate goal of this effort is to integrate the OpenBEMT/ANOPP2 combination into a larger UAM optimization case, where additional disciplines (trajectory, power generation, vehicle weight, etc.) are included. This will require the 


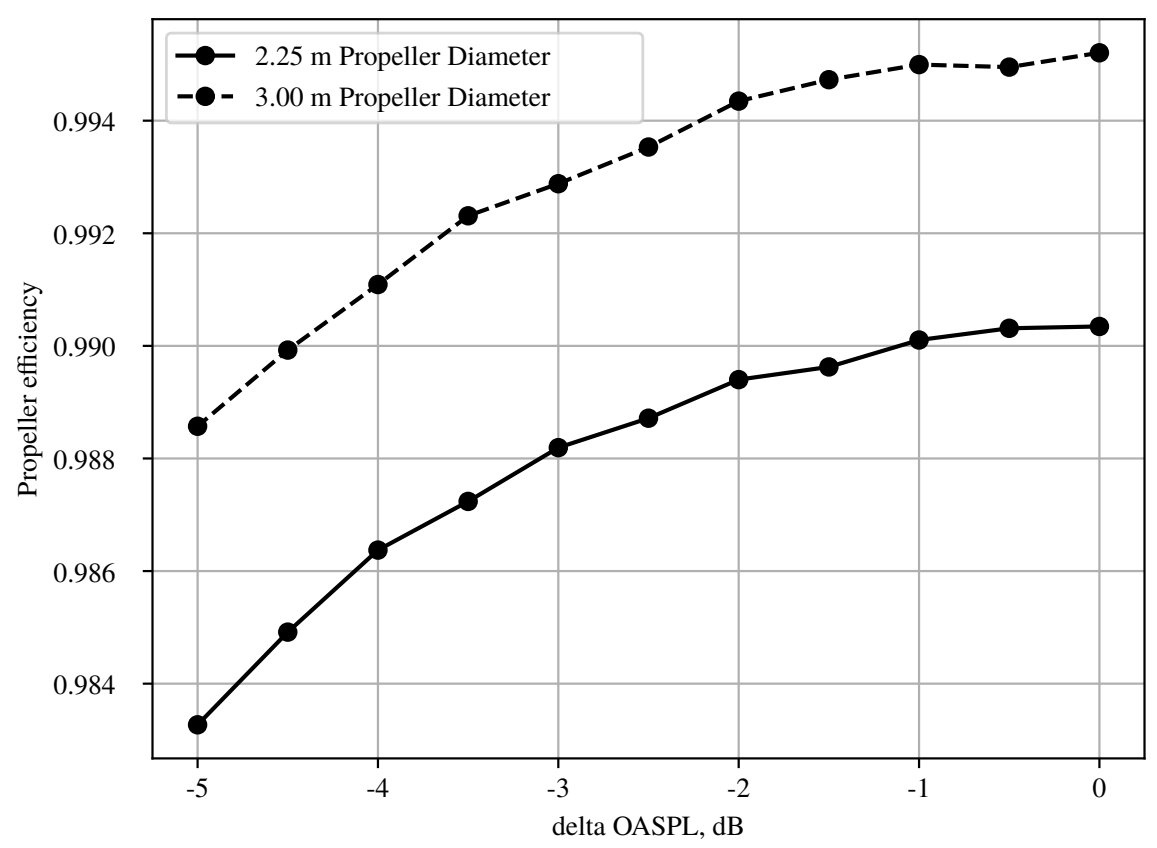

Fig. 10 Relationship between the noise constraint and efficiency objective for case 2, showing the effect of increasing the propeller diameter limit.

OpenBEMT-ANOPP2 toolchain to evaluate the propeller's performance and noise at multiple trajectory points, and record the ANOPP2 acoustic predictions experienced by one or more stationary acoustic observers.

\section{Acknowledgements}

The authors thank Christopher J. Miller of NASA Glenn for the helpful discussions, and reviewing an early form of this work. This work was supported by NASA's Transformational Tools and Technologies (TTT) Project.

\section{References}

[1] Miller, C. J., and Sullivan, J. P., "Noise constraints effecting optimal propeller designs," Tech. Rep. NASA-TM-86967, NASA Lewis Research Center, 01 1985. URL https://ntrs.nasa.gov/search.jsp?R=19850011613 soc. of Automotive Engr. Gen. Aviation Aircraft Meeting and Exposition; 16-19 Apr. 1985; Wichita, KS; United States.

[2] Gur, O., and Rosen, A., "Multidisciplinary Design Optimization of a Quiet Propeller," 14th AIAA/CEAS Aeroacoustics Conference (29th AIAA Aeroacoustics Conference), American Institute of Aeronautics and Astronautics, 2008. doi:10.2514/6.2008-3073, URL http://dx.doi.org/10.2514/6.2008-3073

[3] Gur, O., and Rosen, A., "Optimization of Propeller Based Propulsion System," Journal of Aircraft, Vol. 46, No. 1, 2009 , p. 95-106. doi:10.2514/1.36055, URL http://dx.doi.org/10.2514/1.36055

[4] Pagano, A., Barbarino, M., Casalino, D., and Federico, L., "Tonal and Broadband Noise Calculations for Aeroacoustic Optimization of Propeller Blades in a Pusher Configuration," 15th AIAA/CEAS Aeroacoustics Conference (30th AIAA Aeroacoustics Conference), American Institute of Aeronautics and Astronautics, 2009. doi:10.2514/6.2009-3138.

[5] Pagano, A., Barbarino, M., Casalino, D., and Federico, L., "Tonal and Broadband Noise Calculations for Aeroacoustic Optimization of a Pusher Propeller," Journal of Aircraft, Vol. 47, No. 3, 2010, p. 835-848. doi:10.2514/1.45315, URL http://dx.doi.org/10.2514/1.45315 
[6] Wisniewski, C. F., Byerley, A. R., Heiser, W., Van Treuren, K. W., and Liller, T., "Designing Small Propellers for Optimum Efficiency and Low Noise Footprint," 33rd AIAA Applied Aerodynamics Conference, American Institute of Aeronautics and Astronautics, 2015. doi:10.2514/6.2015-2267, URL http://dx.doi.org/10.2514/6.2015-2267

[7] Lopes, L. V., and Burley, C. L., “ANOPP2 User's Manual: Version 1.2,” Tech. Rep. NASA TM-2016-219342, NASA Langley Research Center, Hampton, VA, United States, 10 2016. URL https://ntrs . nasa.gov/search. jsp?R=20160014858.

[8] Gray, J., Moore, K., and Naylor, B., "OpenMDAO: An Open Source Framework for Multidisciplinary Analysis and Optimization," 13th AIAA/ISSMO Multidisciplinary Analysis Optimization Conference, American Institute of Aeronautics and Astronautics, 2010. doi:10.2514/6.2010-9101, URL http://dx.doi.org/10.2514/6.2010-9101

[9] Hwang, J. T., and Ning, A., "Large-scale multidisciplinary optimization of an electric aircraft for on-demand mobility," 2018 AIAA/ASCE/AHS/ASC Structures, Structural Dynamics, and Materials Conference, American Institute of Aeronautics and Astronautics, 2018. doi:10.2514/6.2018-1384.

[10] Viterna, L. A., and Janetzke, D. C., “Theoretical and experimental power from large horizontal-axis wind turbines,” Tech. Rep. NASA-TM-82944, NASA Lewis Research Center, 09 1982. URL https://ntrs.nasa.gov/search.j jp?R=19820025954

[11] Hwang, J. T., and Martins, J. R., “A fast-prediction surrogate model for large datasets,” Aerospace Science and Technology, Vol. 75, 2018, p. 74-87. doi:10.1016/j.ast.2017.12.030, URL http://dx.doi.org/10.1016/j .ast.2017.12.030

[12] Andrew Ning, S., "A simple solution method for the blade element momentum equations with guaranteed convergence," Wind Energy, 2013, p. n/a-n/a. doi:10.1002/we.1636, URL http://dx.doi.org/10.1002/we.1636

[13] "Aeroacoustics of Flight Vehicles: Theory and Practice. Volume 1: Noise Sources," Tech. Rep. NASA Reference Publication 1258, Vol. 1, NASA Langley Research Center, aug 1991.

[14] Farassat, F., "Linear Acoustic Formulas for Calculation of Rotating Blade Noise," AIAA Journal, Vol. 19, No. 9, 1981, pp. 1122-1130. doi:10.2514/3.60051.

[15] Farassat, F., "Derivation of Formulations 1 and 1A of Farassat," Tech. Rep. NASA/TM 2007-214853, NASA Langley Research Center, Hampton, VA, United States, mar 2007.

[16] Ffowcs Williams, J. E., and Hawkings, D. L., "Sound Generation by Turbulence and Surfaces in Arbitrary Motion," Philosophical Transactions of the Royal Society of London. Series A, Mathematical and Physical Sciences, Vol. 264, No. 1151, 1969, pp. 321-342.

[17] Lopes, L. V., “Compact Assumption Applied to Monopole Term of Farassat's Formulations,” Journal of Aircraft, Vol. 54, No. 5, 2017, p. 1649-1663. doi:10.2514/1.c034048, URL http://dx.doi.org/10.2514/1.CQ34048

[18] Hwang, J. T., "A modular approach to large-scale design optimization of aerospace systems," Ph.D. thesis, University of Michigan, 2015.

[19] Gill, P., Murray, W., and Saunders, M., "SNOPT: An SQP Algorithm for Large-Scale Constrained Optimization,” SIAM Review, Vol. 47, No. 1, 2005, pp. 99-131. doi:10.1137/S0036144504446096, URL https://doi.org/10.1137/ S0036144504446096. 